University of Nebraska - Lincoln

DigitalCommons@University of Nebraska - Lincoln

Norman R. Simon Papers

Research Papers in Physics and Astronomy

8-1987

ON THE INVERSION OF THE BAADE-WESSELINK TECHNIQUE

Norman R. Simon

University of Nebraska - Lincoln, nsimon@unl.edu

Follow this and additional works at: https://digitalcommons.unl.edu/physicssimon

Simon, Norman R., "ON THE INVERSION OF THE BAADE-WESSELINK TECHNIQUE" (1987). Norman $R$. Simon Papers. 64.

https://digitalcommons.unl.edu/physicssimon/64

This Article is brought to you for free and open access by the Research Papers in Physics and Astronomy at DigitalCommons@University of Nebraska - Lincoln. It has been accepted for inclusion in Norman R. Simon Papers by an authorized administrator of DigitalCommons@University of Nebraska - Lincoln. 


\title{
ON THE INVERSION OF THE BAADE-WESSELINK TECHNIQUE
}

\author{
NORMAN R. SIMON \\ Department of Physics and Astronomy, University of Nebraska-Lincoln, Lincoln, Nebraska 68588-0111 \\ Received 1987 March 20, revised 1987 May 23
}

\begin{abstract}
A general framework is given for the inversion of a "two-point" formulation of the Baade-Wesselink technique. This means that phases of equal radius are selected and used to get information regarding brightness and temperature. The inversion method is used to evaluate the surface brightness/(V-R) relation in two classical Cepheids, V482 Sco and R TrA. The slope found is consistent with previously reported values, but it is argued that the radius determinations will be very uncertain. Finally, a proposal is made for employing the B-W inversion to construct a parameterization for brightness and temperature. This leads to the determination of radii and, ultimately, of temperatures and luminosities as well.
\end{abstract}

Key words: stars: pulsation-stars: Cepheids

\section{Introduction}

The determination of the temperatures and radii of pulsating stars has an important impact on many areas in astronomy, including stellar evolution, galactic morphology and history, and the cosmic distance scale. Traditionally, temperatures are obtained by the fitting of static model atmospheres to observed fluxes, while radii are determined using the Baade-Wesselink (B-W) technique.

The B-W technique subsumes a group of related methods all of which rely on the fact that the instantaneous radial displacement of a pulsating star may be obtained by an integration of its observed velocity curve. Once this is done, the radius may be determined provided that one can measure or infer the instantaneous temperature or some related quantity.

A comprehensive critique of the B-W method has been given recently by Gautschy (1986). Early formulations of this technique involved pairs of phases for which the temperatures or surface brightnesses were supposed to be equal. If this condition is satisfied, then each pair yields a radius. Later, these "two-point" methods gave way to more proper statistical formulations which employ the whole of the cycle. However, the latter realizations also require an assumed or derived relation between surface brightness and color. Unfortunately, these pivotal relations have not been tested for pulsating stars except a posteriori via a somewhat circular argument which requires the use of mean radii which have themselves been obtained from a surface brightness-color relation (see, e.g., Barnes and Moffett 1985).

In the present work we return to a two-point formulation which, however, takes note of the following, very interesting, circumstance: while we are still not capable of selecting phases of equal surface brightness, modern observational techniques provide the means for determining, with high accuracy, pairs of phases of equal radius. Thus, in what follows, we shall propose an inversion of the B-W method whereby information on the instantaneous radius is used to learn something about the surface brightness or temperature. In Section II we provide a general framework for this proposal which is then employed in Section III to examine the relationship between surface brightness and color in two classical Cepheids. Finally, in Section IV we suggest how the inversion technique may be employed to construct a calibration which leads ultimately to the determination of radii, temperatures, and luminosities.

\section{The Pulsating Star at Phases of Equal Displacement}

Let us write for the radial velocity of a pulsating star

$$
v_{r}=A_{0}-A_{j} \sin \left[j \omega\left(t-t_{0}\right)+\phi_{j}\right],
$$

where a summation convention is understood. The coefficients $A_{0},\left(A_{j}, \phi_{j}\right)$ may be determined by fitting the observed radial-velocity curve with a Fourier series. For classical Cepheids four to eight terms will typically suffice (Simon and Teays 1983). The pulsational velocity $v$ is then given by use of a projection factor $f$, viz.,

$$
v=f\left(v_{r}-A_{0}\right) .
$$

We then obtain the instantaneous displacement by integration

$$
r=R-R_{0}=\frac{f}{j \omega} A_{j} \cos \left[j \omega\left(t-t_{0}\right)+\phi_{j}\right]
$$

and the acceleration by differentiation: 


$$
a=-j \omega f A_{j} \cos \left[j \omega\left(t-t_{0}\right)+\phi_{\mathrm{j}}\right] .
$$

Let us now consider the ratio of the instantaneous radii at two phases of the pulsation

$$
\alpha \equiv \frac{R_{1}}{R_{2}}=\frac{\left(R_{0}+r_{1}\right)}{\left(R_{0}+r_{2}\right)} .
$$

For $r_{1} / R_{0}, r_{2} / R_{0}<<1$, we may expand equation (4) to obtain

$$
\alpha=1+\frac{1}{R_{0}}\left(r_{1}-r_{2}\right) .
$$

Suppose we choose phases such that the displacements are equal, i.e., $r_{1}=r_{2}$ as determined from equation (2). In that case the quantity $\alpha$ is ostensibly unity according to equation (5). However, the real value of $\alpha$ is

$$
\alpha=1+\frac{1}{R_{0}}\left(\delta r_{1}-\delta r_{2}\right),
$$

where $\delta r_{1}$ and $\delta r_{2}$ are observational errors in the determination of the displacements. (For the moment we ignore the question of the projection factor $f$.) If one can measure the radial velocity at a given phase with some error of magnitude $|\delta v|$ then the error in the displacement at that phase will be approximately $|\delta r|=|\delta v| / \omega$. Then, assuming the worst case in equation (6), i.e., that the errors add, we obtain

$$
1-\epsilon \lesssim \alpha \lesssim 1+\epsilon
$$

where

$$
\epsilon=\frac{2|\delta v|}{\omega R_{0}} .
$$

Now; of course, the quantity $\alpha$ may also be determined in another way, namely,

$$
\tilde{\alpha}=\frac{\tilde{R}_{1}}{\tilde{R}_{2}}
$$

where

$$
\tilde{R}_{1}^{2}=\frac{1}{a c \pi} \frac{L_{1}}{T_{1}^{4}}, \quad \tilde{R}_{2}^{2}=\frac{1}{a c \pi} \frac{L_{2}}{T_{2}^{4}} .
$$

Here and in what follows, the tilde will denote radial displacements determined via luminosity and temperature, while symbols lacking the tilde indicate, where appropriate, that displacements are obtained directly from the velocity curve.

Let us imagine, for a moment, that energy scans at many wavelengths, extending into the ultraviolet and infrared, enable us to measure, with very little error, the ratio of the luminosities at a pair of phases. Then, from equations (9) and (10), one sees that the error in measuring $\tilde{\alpha}$ will be given by

$$
\frac{\delta \tilde{\alpha}}{\tilde{\alpha}}=2 \theta
$$

where

$$
\theta=\frac{\delta T_{2}}{T_{2}}-\frac{\delta T_{1}}{T_{1}},
$$

and we have assumed that the errors, $\delta T$, in temperature measurement are small with respect to $T$.

We now define

$$
\beta=|\tilde{\alpha}-1|-\epsilon .
$$

Suppose for a given star we accumulate a set of pairs of phases of equal displacement according to equation (2). For the purposes of illustration we select two of these pairs-a "good" pair which we call pair $a$ and a "bad" pair labeled pair $b$.

Pair $a$ is called good because for these points we find $\beta$ $\leq 0$. This means that the value $\tilde{\alpha}$ obtained from measuring the temperature (eqs. (9) and (10)) agrees with the value of $\alpha$ calculated by integration of the velocity curve to within the error bars on the latter, given by equation (7). In that case it is easy to show that

$$
|\theta| \leq \frac{1}{2 \tilde{\alpha}}[2 \epsilon+\beta], \quad(\beta \leq 0) .
$$

To make our illustration more concrete let us estimate the error $\epsilon$ for a typical classical Cepheid. We take

$$
P=2 \pi / \omega=5 d, \quad|\delta v|=2 \mathrm{~km} \mathrm{sec}^{-1}, \quad R_{0}=40 R_{\odot},
$$

where the value for $|\delta v|$ comes from Gieren (1985). Putting these together in equation (8) yields

$$
\epsilon \simeq 1.0 \times 10^{-2} .
$$

Since, for the good pair $a$, one has $\beta \leq 0$, it can be seen from equations (13) and (14) that $\tilde{\alpha} \simeq 1$, and then from equations (14) and (12) that

$$
\left|\frac{\delta T_{2}}{T_{2}}-\frac{\delta T_{1}}{T_{1}}\right| \leq 10^{-2} .
$$

For $T=5500 \mathrm{~K}$, this implies that the error in measuring the temperatures must have been $\delta T \leq 55 \mathrm{~K}$ ! Of course it might be argued that the individual errors, $\delta T_{1}$ and $\delta T_{2}$, could have been much larger, satisfying equation (16) by cancellation. However, this does not seem likely. As shall be illustrated specifically in the next section, two points of equal displacement will, in general, correspond to two pulsation phases with very different hydrodynamic motions, and thus to static model atmospheres with different gravities. Thus it does not appear very probable that a common systematic error of, say, hundreds of degrees is responsible for a cancellation which produces a net error of only $50 \mathrm{~K}$. It is much more likely that, for the good pair $a$, the static atmosphere approximation is rather accurate, yielding temperatures which are not too far off the mark.

We may contrast this with the situation for the bad pair 
$b$ for which $\tilde{\alpha}$ does not agree with $\alpha$ to within the errors on the latter. In this case $\beta>0$ and it can be shown from equations (7), (11), and (13) that

$$
\frac{\beta}{2 \tilde{\alpha}} \leq|\theta| \leq \frac{1}{2 \tilde{\alpha}}(2 \epsilon+\beta) \quad(\beta>0) .
$$

Thus we are provided both maximum and minimum errors on $|\theta|$ and hence on the relative temperatures. If $\beta$ is very large, the pair $b$ will be bad indeed.

Let us now summarize our development so far as follows. Presently available, highly accurate observations of radial velocity make it possible to determine pairs of phases of equal displacement with a small error given by equations (7) and (8). This, in turn, affords a check on the relative accuracy of the temperatures measured at the two phases, provided that the ratio of their luminosities can be found with negligible uncertainty. In this way it becomes possible to build up a set of phase pairs for which the temperatures seem consistent (and thus for which the static atmosphere approximation is probably not too bad) as well as a set of pairs for which inconsistency in the temperatures indicates a problem in their determination. This information could then be used as a basis for guiding the formulation of atmospheric models appropriate for the dynamics of a pulsating star. We shall return to this question in Section IV.

We close the present section by returning to a problem that we set aside above, namely that of the possible errors introduced into the measured displacements by uncertainties in the projection factor $f$. It is clear from equation (5) that the projection factor will not affect the equality $r_{1}$ $=r_{2}$ provided that $f$ is constant over the pulsation cycle. However, in a recent paper Hindsley and Bell (1986) have suggested that $f$ is actually variable. The numbers they give indicate a range of the order $\Delta f=0.04$. Assuming the worst case in equation (5), namely that phases 1 and 2 fall on opposite ends of this range, and taking $\left(r_{1}+r_{2}\right) / R_{0}=$ 0.1 we obtain for the error in $\alpha$ due to the projection factor, $\epsilon^{\prime} \simeq 0.004$. Comparing this to the value given by equation (15), one sees that possible uncertainties in $f$ reduce the absolute precision of our technique only slightly. The same argument may be made regarding the error in the expansion in equation (5) and similar expansions to be performed later. Henceforth we shall ignore all these errors.

\section{Check on the Surface-Brightness Method}

In the previous section we have indicated how the B-W technique might be inverted to give information on the Cepheid temperature calibration provided that the ratio of the luminosities at two phases of equal displacement could be measured very accurately. However, to obtain the luminosity ratio it would be necessary to make not only optical but also ultraviolet and infrared measurements and to perform the delicate task of mutual calibra- tion of the data. Because such observations do not yet exist, we wish in the present section to show how the inversion technique may be employed with existing data as a check on radius determinations.

We begin by writing equations (9) and (10) in the form

$$
\log \tilde{\alpha}=\frac{V_{2}-V_{1}}{5}+\frac{(\mathrm{BC})_{2}-(\mathrm{BC})_{1}}{5}+2\left(\log T_{2}-\log T_{1}\right)
$$

where $\mathrm{BC}$ is the bolometric correction, defined by

$$
M_{\mathrm{BOL}}=M_{V}+\mathrm{BC} \text {. }
$$

Let us now make the standard assumption that both $\log T$ and BC are linear functions of some color which we will choose to be $(V-R)$. Then

$$
\log \tilde{\alpha}=2\left\{0.1\left(V_{2}-V_{1}\right)-b\left[(V-R)_{2}-(V-R)_{1}\right]\right\} .
$$

Equation (17), of course, has a familiar form, reminiscent of the relations which comprise the surface-brightness technique of Barnes et al. (1977). These relations have been given as follows by Gieren (1985), according to the calibration by Barnes (1980):

$$
\begin{gathered}
F_{V}=4.2207-0.1 V_{0}-0.5 \log \phi \\
F_{V}=3.956-b(V-R)_{0} \\
D_{0}+\Delta D=10^{-3} r \phi .
\end{gathered}
$$

Here $F_{V}$ is the surface brightness parameter, $V_{0}$ and $(V-R)_{0}$ are unreddened magnitudes, $D_{0}$ and $\Delta D$ are the average diameter and instantaneous diameter perturbation, respectively, $r$ is the distance to the pulsating star, and $\phi$ is the instantaneous angular diameter. The coefficient $b$ in equation (18) is given specifically by Gieren (1985): $b=0.363$.

It is easy to combine the above equations to form with regard to a pair of phases the quantity

$$
\begin{aligned}
\tilde{\eta} & \equiv \log \left(\phi_{1} / \phi_{2}\right) \\
& =2\left\{0.1\left(V_{2}-V_{1}\right)-b\left[(V-R)_{2}-(V-R)_{1}\right]\right\} .
\end{aligned}
$$

Comparing equations (17) and (20) we see that

$$
\tilde{\eta}=\log \tilde{\alpha},
$$

while equation (20) shows us that for phases of equal displacement we must have $\tilde{\eta}=0$. We note that both equations (17) and (20) contain raw magnitudes. No dereddening is necessary so long as the reddening law itself does not have a significant dependence on color.

Now

$$
\frac{\phi_{1}}{\phi_{2}}=\frac{D_{0}+\Delta D_{1}}{D_{0}+\Delta D_{2}} \simeq 1+\frac{1}{R_{0}}\left(r_{1}-r_{2}\right)
$$

a relation identical to equation (5). Let us define the quantity

$$
\eta=\log \left(\phi_{1} / \phi_{2}\right)
$$


where the lack of a tilde again indicates a determination via the velocity curve.

We may now argue exactly as above to show that

$$
1-\epsilon \lesssim \frac{\phi_{1}}{\phi_{2}} \lesssim 1+\epsilon
$$

and hence

$$
-0.004 \lesssim \eta \lesssim 0.004,
$$

where we have used the value of $\epsilon$ from equation (15). Thus for pairs of points with ostensibly equal displacements, the value of $\eta$ (ostensibly zero) actually lies within the limits given by equation (22).

We are now ready to apply our methodology to a set of observations. For this purpose we choose two short-period Cepheids, V482 Scorpii $(P=4$.53) and $\mathrm{R}$ Trianguli Australis ( $P=3.39$ ), both of which were observed simultaneously in radial velocity and light by Gieren $(1981 a, b)$. Fourier fits to the radial-velocity curves of these stars have already been given by Simon and Teays (1983). We now supplement these with fourth-order fits to the published $V$ magnitudes and $(V-R)$ colors (Gieren $1981 b)$. The Fourier series in this case have the form $A_{0}+A_{j}$ $\cos \left[j \omega\left(t-t_{0}\right)+\phi_{j}\right]$ with $t=$ HJD -2444000 and $t_{0}=$ 421.0. This phasing is the same as for the velocity fits in Simon and Teays (1983). The Fourier coefficients for $V$ and $(V-R)$ are given in Table $\mathrm{I}$.

We shall now proceed by choosing a set of pairs of phases of equal displacement as determined by equation (2) and then using the $V$ and $(V-R)$ fits in equation (20) to calculate the quantity $\tilde{\eta}$ for each pair. To do this we must specify a value for the surface-brightness coefficient $b$. We shall choose at the outset $b=0.363$ (Barnes 1980) although it would not be surprising if this value needed adjustment, since the $(V-R)$ data we are treating are on the Cousins system while the coefficient was determined from Johnson photometry. In Tables II and III we present the results for V482 Sco and $\mathrm{R} T \mathrm{TA}$, respectively. The first six columns give, in order, a bookkeeping number assigned to each pair, and then the radial displacement, pulsational velocity, acceleration, $V$ magnitude, and $(V-R)$ color for each phase. The next-to-last column shows $\tilde{\eta}$ as obtained from equation (20) using the value $b$ $=0.363$. It is also possible to formally assign for each pair a value of $b$ which will make the right-hand side (RHS) of equation (20) vanish as it ought to. We denote this value by $b^{\prime}$ and list it in the last column of Tables II and III.

It is easy to see that, for most of the pairs, the value of $\tilde{\eta}$ obtained from the Johnson surface-brightness calibration lies far outside the error bars given in equation (22). These discrepancies are clearly systematic $(\tilde{\eta}<0$ in all cases) and are very large. To demonstrate this let us use equation (21) to define

$$
\begin{aligned}
\eta_{\mathrm{m}}(r) & \equiv \log \left(\frac{\phi_{\mathrm{m}}}{\phi}\right) \\
& =\log \left[1+\frac{1}{R_{0}}\left(r_{\mathrm{m}}-r\right)\right] \simeq \frac{\log e}{R_{0}}\left(r_{\mathrm{m}}-r\right)
\end{aligned}
$$

where $r$ and $\phi$ are the radial displacement and angular diameter at an arbitrary phase, while $r_{\mathrm{m}}$ and $\phi_{\mathrm{m}}$ refer to the phase of maximum radius. It is clear from equation (23) that as $r$ ranges from maximum to minimum displacement, $\eta_{\mathrm{m}}(r)$ must vary from zero to its maximum value. Then, during the other half of the cycle (expansion phase),

TABLE I

Fourier Decomposition Coefficients

$\left(\mathrm{T}=\mathrm{HJD}-2444000, \mathrm{~T}_{\mathrm{O}}=421.0\right)$

\begin{tabular}{lccccccccc}
\hline Color & $\mathrm{A}_{0}$ & $\mathrm{~A}_{1}$ & $\mathrm{~A}_{2}$ & $\mathrm{~A}_{3}$ & $\mathrm{~A}_{4}$ & $\phi_{1}$ & $\phi_{2}$ & $\phi_{3}$ & $\phi_{4}$ \\
\hline \multicolumn{7}{c}{$\mathrm{V}$ V82 Sco } \\
\hline $\mathrm{V}$ & 7.997 & 0.280 & 0.099 & 0.037 & 0.012 & 1.12 & 0.231 & 5.78 & 4.90 \\
\hline $\mathrm{V}-\mathrm{R}$ & 0.586 & 0.063 & 0.023 & 0.010 & 0.001 & 1.39 & 0.228 & 5.74 & 0.556 \\
\hline & & & & $\mathrm{R}$ & $\mathrm{TrA}$ & & & & \\
\hline $\mathrm{V}$ & 6.669 & 0.249 & 0.075 & 0.028 & 0.011 & 3.22 & 4.29 & 5.42 & 0.287 \\
\hline $\mathrm{V}-\mathrm{R}$ & 0.413 & 0.055 & 0.019 & 0.007 & 0.004 & 3.50 & 4.39 & 5.53 & 1.00 \\
\hline \hline
\end{tabular}




\begin{tabular}{|c|c|c|c|c|c|c|c|}
\hline $\begin{array}{l}\text { Pair } \\
\text { No. }\end{array}$ & $r$ & $\mathrm{v}$ & a & V & $V-R$ & $n$ & $b^{\prime}$ \\
\hline \multirow[t]{2}{*}{1} & 1.606 & $3.97(-1)$ & $2.06(-4)$ & 8.046 & 0.610 & $-1.04(-4)$ & 0.385 \\
\hline & 1.606 & $-4.00(-1)$ & $2.23(-4)$ & 8.037 & 0.608 & & \\
\hline \multirow[t]{2}{*}{2} & 1.519 & 4.40 & $1.47(-4)$ & 8.098 & 0.625 & $-2.76(-3)$ & 0.411 \\
\hline & 1.519 & -5.73 & $2.82(-4)$ & 7.980 & 0.596 & & \\
\hline \multirow[t]{2}{*}{3} & 1.362 & 7.44 & $1.86(-4)$ & 8.136 & 0.631 & $-9.12(-3)$ & 0.469 \\
\hline & 1.361 & -9.62 & $2.52(-4)$ & 7.934 & 0.588 & & \\
\hline \multirow[t]{2}{*}{4} & 1.209 & 9.92 & $2.15(-4)$ & 8.161 & 0.632 & $-1.55(-2)$ & 0.520 \\
\hline & 1.211 & $-1.19(+1)$ & $2.05(-4)$ & 7.903 & 0.582 & & \\
\hline \multirow[t]{2}{*}{5} & 1.053 & $1.21(+1)$ & $2.16(-4)$ & 8.180 & 0.631 & $-2.10(-2)$ & 0.554 \\
\hline & 1.051 & $-1 \cdot 35(+1)$ & $1.58(-4)$ & 7.876 & 0.576 & & \\
\hline \multirow[t]{2}{*}{6} & $7 \cdot 163(-1)$ & $1.54(+1)$ & $1.63(-4)$ & 8.208 & 0.629 & $-2.73(-2)$ & 0.566 \\
\hline & $7 \cdot 177(-1)$ & $-1 \cdot 55(+1)$ & $1.13(-4)$ & 7.827 & 0.562 & & \\
\hline \multirow[t]{2}{*}{7} & $4.054(-1)$ & $1 \cdot 72(+1)$ & $9.96(-5)$ & 8.226 & 0.629 & $-2.84(-2)$ & 0.534 \\
\hline & $4.031(-1)$ & $-1 \cdot 72(+1)$ & $1.37(-4)$ & 7.784 & 0.547 & & \\
\hline \multirow[t]{2}{*}{8} & $-2.408(-3)$ & $1.83(+1)$ & $5.29(-5)$ & 8.248 & 0.633 & $-2.70(-2)$ & 0.491 \\
\hline & $-2.205(-3)$ & $-1 \cdot 97(+1)$ & $1.92(-4)$ & 7.729 & 0.527 & & \\
\hline \multirow[t]{2}{*}{9} & $-3.038(-1)$ & $1.89(+1)$ & $5.17(-5)$ & 8.263 & 0.636 & $-2.61(-2)$ & 0.470 \\
\hline & $-3.033(-1)$ & $-2 \cdot 17(+1)$ & $1.97(-4)$ & 7.691 & 0.515 & & \\
\hline \multirow[t]{2}{*}{10} & $-5.605(-1)$ & $1.94(+1)$ & $5.76(-5)$ & 8.273 & 0.639 & $-2.60(-2)$ & 0.461 \\
\hline & $-5.609(-1)$ & $-2 \cdot 32(+1)$ & $1.59(-4)$ & 7.664 & 0.507 & & \\
\hline \multirow[t]{2}{*}{11} & -1.009 & $2.02(+1)$ & $2.51(-5)$ & 8.273 & 0.637 & $-2.68(-2)$ & 0.459 \\
\hline & -1.009 & $-2.44(+1)$ & $3.19(-8)$ & 7.634 & 0.498 & & \\
\hline \multirow[t]{2}{*}{12} & $-1 \cdot 359$ & $1.99(+1)$ & $8.00(-5)$ & 8.247 & 0.628 & $-2 \cdot 70(-2)$ & 0.465 \\
\hline & $-1 \cdot 359$ & $-2.34(+1)$ & $-1 \cdot 92(-4)$ & 7.632 & 0.561 & & \\
\hline \multirow[t]{2}{*}{13} & -1.863 & $1.56(+1)$ & $-3.91(-4)$ & 8.139 & 0.600 & $-2.18(-2)$ & 0.479 \\
\hline & -1.863 & $-1 \cdot 71(+1)$ & $-5.41(-4)$ & 7.689 & 0.506 & & \\
\hline \multirow[t]{2}{*}{14} & -2.071 & $1.01(+1)$ & $-5.84(-4)$ & 8.043 & 0.578 & $-1.43(-2)$ & 0.486 \\
\hline & -2.071 & $-1.07(+1)$ & $-6.91(-4)$ & 7.760 & 0.520 & & \\
\hline \multirow[t]{2}{*}{15} & -2.183 & $5.10(-1)$ & $-7 \cdot 34(-4)$ & 7.901 & 0.548 & $-7.05(-4)$ & 0.489 \\
\hline & -2.183 & $-5.00(-1)$ & $-7.40(-4)$ & 7.887 & 0.545 & & \\
\hline
\end{tabular}

$\eta_{m}$ will reverse this variation. Furthermore, $\eta_{m}$ is always nonnegative.

In Figure 1 we plot the corresponding quantity

$$
\tilde{\eta}_{\mathrm{m}}=2\left\{0.1\left(V-V_{\mathrm{m}}\right)-b\left[(V-R)-(V-R)_{\mathrm{m}}\right]\right\}
$$

versus radial displacement $r$ for $\mathrm{V} 482$ Sco and $\mathrm{R} \operatorname{TrA}$. Here $V_{\mathrm{m}}$ and $(V-R)_{\mathrm{m}}$ are the $V$ magnitude and $(V-R)$ color at maximum displacement. The value of $b$ is that already used above, $b=0.363$. The expansion part of the cycle is rendered by a solid curve, while the dashed curve denotes contraction. Arrows indicate the direction around the cycle. The phases of maximum and minimum brightness in the $V$ magnitude are shown by vertical bars.

We may note from Figure 1 a number of serious contradictions for both stars. First, the maximum of $\tilde{\eta}_{m}$ comes substantially earlier than minimum displacement. Second, over a good portion of the contraction phase, $\tilde{\eta}_{m}$ becomes negative. And, third, the loops are very fat, meaning that at virtually any given displacement $r$, the value of $\tilde{\eta}_{m}$ is very different for contraction and expansion. The magnitude of this last difficulty is indicated by the vertical error bars we have placed inside the loops.
These show the spread that would be introduced into the loop by either maximal errors in determining $\eta$ from the velocity curve (see eq. (22)) or by maximal observational uncertainty in determining $(V-R)$ colors for the given pair.

Returring now to Tables II and III, we notice that the value of $b^{\prime}$ is in all cases greater than 0.363 . Thus we might hope to obtain some overall improvement by choosing a new, larger value for $b$. We make no attempt here to carefully select a value which minimizes the discrepancies (this subject will be discussed below) but instead arbitrarily choose $b=0.5$ merely to demonstrate the effect. Table IV recompiles for both stars the set of points of equal displacement and lists the values of $\tilde{\eta}$ for both the old and new values of $b$. We see from the last column of Table IV that the discrepancy has indeed been narrowed, in most cases to a value below the spread indicated by equation (22).

This improvement may also be noted in Figure 2 where we have replotted the $\tilde{\eta}_{\mathrm{m}}$ vs. $r$ graphs, this time using $b=$ 0.5. We see that for both stars (1) $\tilde{\eta}_{m}$ now attains its maximum near minimum displacement; (2) the domain of 
TABLE III

Points of Equal Displacement for $\mathrm{R} \operatorname{Tr} A$

\begin{tabular}{|c|c|c|c|c|c|c|c|}
\hline $\begin{array}{l}\text { Pair } \\
\text { No. }\end{array}$ & $r$ & $\mathrm{v}$ & $a$ & V & $V-R$ & $n$ & $b^{\prime}$ \\
\hline 1 & $\begin{array}{l}7.834(-1) \\
7.834(-1)\end{array}$ & $\begin{array}{r}5.71(-1) \\
-5.55(-1)\end{array}$ & $\begin{array}{l}2.82(-4) \\
2.86(-4)\end{array}$ & $\begin{array}{l}6.722 \\
6.710\end{array}$ & $\begin{array}{l}0.437 \\
0.436\end{array}$ & $-1.53(-3)$ & 0.931 \\
\hline 2 & $\begin{array}{l}7.398(-1) \\
7.400(-1)\end{array}$ & $\begin{array}{r}3.77 \\
-3.97\end{array}$ & $\begin{array}{l}1.79(-4) \\
2.22(-4)\end{array}$ & $\begin{array}{l}6.759 \\
6.663\end{array}$ & $\begin{array}{l}0.441 \\
0.429\end{array}$ & $-1.05(-2)$ & 0.797 \\
\hline 3 & $\begin{array}{l}6.470(-1) \\
6.473(-1)\end{array}$ & $\begin{array}{r}5.09 \\
-6.13\end{array}$ & $\begin{array}{l}1.35(-5) \\
1.25(-4)\end{array}$ & $\begin{array}{l}6.795 \\
6.612\end{array}$ & $\begin{array}{l}0.447 \\
0.418\end{array}$ & $-1.55(-2)$ & 0.630 \\
\hline 4 & $\begin{array}{l}4.850(-1) \\
4.851(-1)\end{array}$ & $\begin{array}{r}4.60 \\
-7.81\end{array}$ & $\begin{array}{l}3.19(-5) \\
1.13(-4)\end{array}$ & $\begin{array}{l}6.852 \\
6.548\end{array}$ & $\begin{array}{l}0.457 \\
0.400\end{array}$ & $-1.95(-2)$ & 0.535 \\
\hline 5 & $\begin{array}{l}3.252(-1) \\
3.249(-1)\end{array}$ & $\begin{array}{r}7.76 \\
-9.80\end{array}$ & $\begin{array}{l}2.91(-4) \\
2.03(-4)\end{array}$ & $\begin{array}{l}6.880 \\
6.499\end{array}$ & $\begin{array}{l}0.455 \\
0.385\end{array}$ & $-2.51(-2)$ & 0.542 \\
\hline 6 & $\begin{array}{l}1.636(-1) \\
1.643(-1)\end{array}$ & $\begin{array}{r}1.17(+1) \\
-1.22(+1)\end{array}$ & $\begin{array}{l}3.53(-4) \\
2.64(-4)\end{array}$ & $\begin{array}{l}6.886 \\
6.457\end{array}$ & $\begin{array}{l}0.453 \\
0.373\end{array}$ & $-2.81(-2)$ & 0.540 \\
\hline 7 & $\begin{array}{l}7.399(-3) \\
8.138(-3)\end{array}$ & $\begin{array}{r}1.45(+1) \\
-1.44(+1)\end{array}$ & $\begin{array}{l}3.08(-4) \\
2.59(-4)\end{array}$ & $\begin{array}{l}6.888 \\
6.420\end{array}$ & $\begin{array}{l}0.452 \\
0.363\end{array}$ & $-2.93(-2)$ & 0.528 \\
\hline 8 & $\begin{array}{l}-1.650(-1) \\
-1.656(-1)\end{array}$ & $\begin{array}{r}1.65(+1) \\
-1.63(+1)\end{array}$ & $\begin{array}{l}2.03(-4) \\
1.92(-4)\end{array}$ & $\begin{array}{l}6.889 \\
6.386\end{array}$ & $\begin{array}{l}0.452 \\
0.354\end{array}$ & $-2.92(-2)$ & 0.511 \\
\hline 9 & $\begin{array}{l}-3.181(-1) \\
-3.164(-1)\end{array}$ & $\begin{array}{r}1.74(+1) \\
-1 \cdot 72(+1)\end{array}$ & $\begin{array}{l}8.52(-5) \\
9.69(-5)\end{array}$ & $\begin{array}{l}6.887 \\
6.364\end{array}$ & $\begin{array}{l}0.452 \\
0.346\end{array}$ & $-2.81(-2)$ & 0.496 \\
\hline 10 & $\begin{array}{l}-4.905(-1) \\
-4.908(-1)\end{array}$ & $\begin{array}{r}1.75(+1) \\
-1.74(+1)\end{array}$ & $\begin{array}{l}-6.02(-5) \\
-4.18(-5)\end{array}$ & $\begin{array}{l}6.880 \\
6.348\end{array}$ & $\begin{array}{l}0.451 \\
0.340\end{array}$ & $-2.57(-2)$ & 0.479 \\
\hline 11 & $\begin{array}{l}-6.554(-1) \\
-6.551(-1)\end{array}$ & $\begin{array}{r}1.66(+1) \\
-1.66(+1)\end{array}$ & $\begin{array}{l}-2.05(-4) \\
-1.89(-4)\end{array}$ & $\begin{array}{l}6.865 \\
6.347\end{array}$ & $\begin{array}{l}0.447 \\
0.336\end{array}$ & $-2.25(-2)$ & 0.464 \\
\hline 12 & $\begin{array}{l}-8.404(-1) \\
-8.408(-1)\end{array}$ & $\begin{array}{r}1.42(+1) \\
-1.43(+1)\end{array}$ & $\begin{array}{l}-3.67(-4) \\
-3.65(-4)\end{array}$ & $\begin{array}{l}6.831 \\
6.370\end{array}$ & $\begin{array}{l}0.438 \\
0.336\end{array}$ & $-1.76(-2)$ & 0.449 \\
\hline 13 & $\begin{array}{l}-9.847(-1) \\
-9.836(-1)\end{array}$ & $\begin{array}{r}1.07 .(+1) \\
-1.08(+1)\end{array}$ & $\begin{array}{l}-4.94(-4) \\
-5.00(-4)\end{array}$ & $\begin{array}{l}6.778 \\
6.417\end{array}$ & $\begin{array}{l}0.424 \\
0.342\end{array}$ & $-1.24(-2)$ & 0.439 \\
\hline 14 & $\begin{array}{l}-1.131 \\
-1.131\end{array}$ & $\begin{array}{r}8 \cdot 32(-1) \\
-8 \cdot 23(-1)\end{array}$ & $\begin{array}{l}-6.27(-4) \\
-6.28(-4)\end{array}$ & $\begin{array}{l}6.612 \\
6.583\end{array}$ & $\begin{array}{l}0.382 \\
0.375\end{array}$ & $-8.87(-4)$ & 0.429 \\
\hline
\end{tabular}
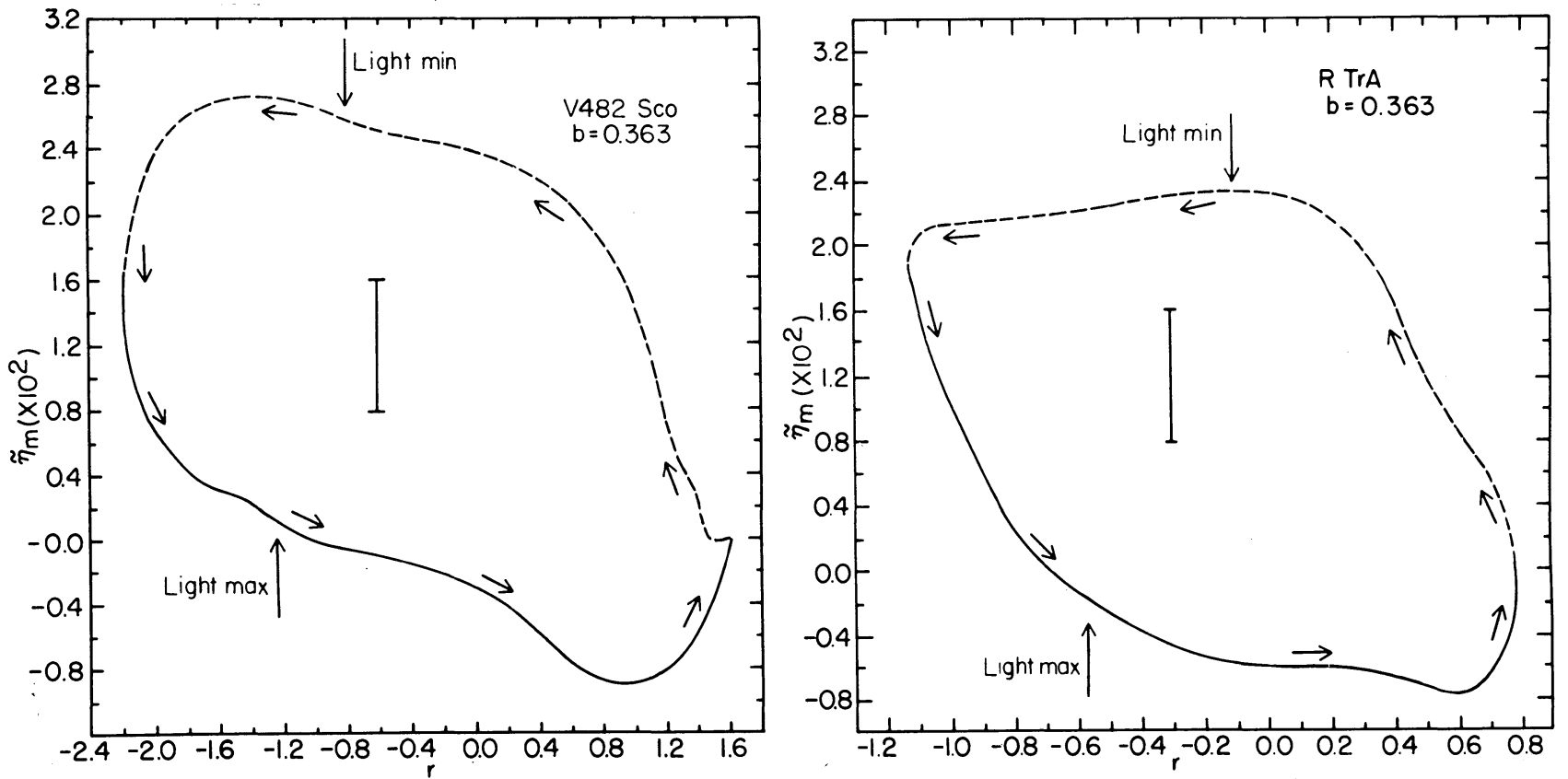

FIG. $1-\tilde{\eta}$ vs. radial displacement $r$ for V482 Sco and R TrA for the case $b=0.363$. Solid curve: expansion; dashed curve: contraction. Arrows indicate direction around cycle. Phases of maximum and minimum light ( $V$ magnitude) are shown. Vertical bars indicate maximal spread due to observational errors. 
TABLE IV

Variation of $\bar{n}$ with the Coefficient $\underline{b}$

\begin{tabular}{|c|c|c|c|c|}
\hline & \multicolumn{2}{|c|}{ V482 Sco } & \multicolumn{2}{|c|}{$\mathrm{R} \operatorname{Tr} \mathrm{A}$} \\
\hline Pair & & & & \\
\hline No. & $b=0.363$ & $b=0.5$ & $b=0.363$ & $b=0.5$ \\
\hline 1 & $-1.04(-4)$ & $5.41(-4)$ & $-1.53(-3)$ & $-1.16(-3)$ \\
\hline 2 & $-2.76(-3)$ & $5.14(-3)$ & $-1.05(-2)$ & $-7.20(-3)$ \\
\hline 3 & $-9.12(-3)$ & $2.66(-3)$ & $-1.55(-2)$ & $-7.56(-3)$ \\
\hline 4 & $-1.55(-2)$ & $-1.93(-3)$ & $-1.95(-2)$ & $-3 \cdot 92(-3)$ \\
\hline 5 & $-2.10(-2)$ & $-5.91(-3)$ & $-2.51(-2)$ & $-5.87(-3)$ \\
\hline 6 & $-2.73(-2)$ & $-8.92(-3)$ & $-2.81(-2)$ & $-6 \cdot 30(-3)$ \\
\hline 7 & $-2.84(-2)$ & $-5.70(-3)$ & $-2.93(-2)$ & $-5.00(-3)$ \\
\hline 8 & $-2.70(-2)$ & $2.00(-3)$ & $-2.92(-2)$ & $-2.24(-3)$ \\
\hline 9 & $-2.61(-2)$ & $7.24(-3)$ & $-2.81(-2)$ & $8.59(-4)$ \\
\hline 10 & $-2.60(-2)$ & $1.02(-2)$ & $-2.57(-2)$ & $4.74(-3)$ \\
\hline 11 & $-2.68(-2)$ & $1.13(-2)$ & $-2.25(-2)$ & $8.11(-3)$ \\
\hline 12 & $-2.70(-2)$ & $9.20(-3)$ & $-1 \cdot 76(-2)$ & $1.06(-2)$ \\
\hline 13 & $-2.18(-2)$ & $3.93(-3)$ & $-1 \cdot 24(-2)$ & $1.01(-2)$ \\
\hline 14 & $-1.43(-2)$ & $1.66(-3)$ & $-8.87(-4)$ & $9.58(-4)$ \\
\hline 15 & $-7.05(-4)$ & $5.92(-5)$ & $\cdots$ & $\cdots$ \\
\hline
\end{tabular}
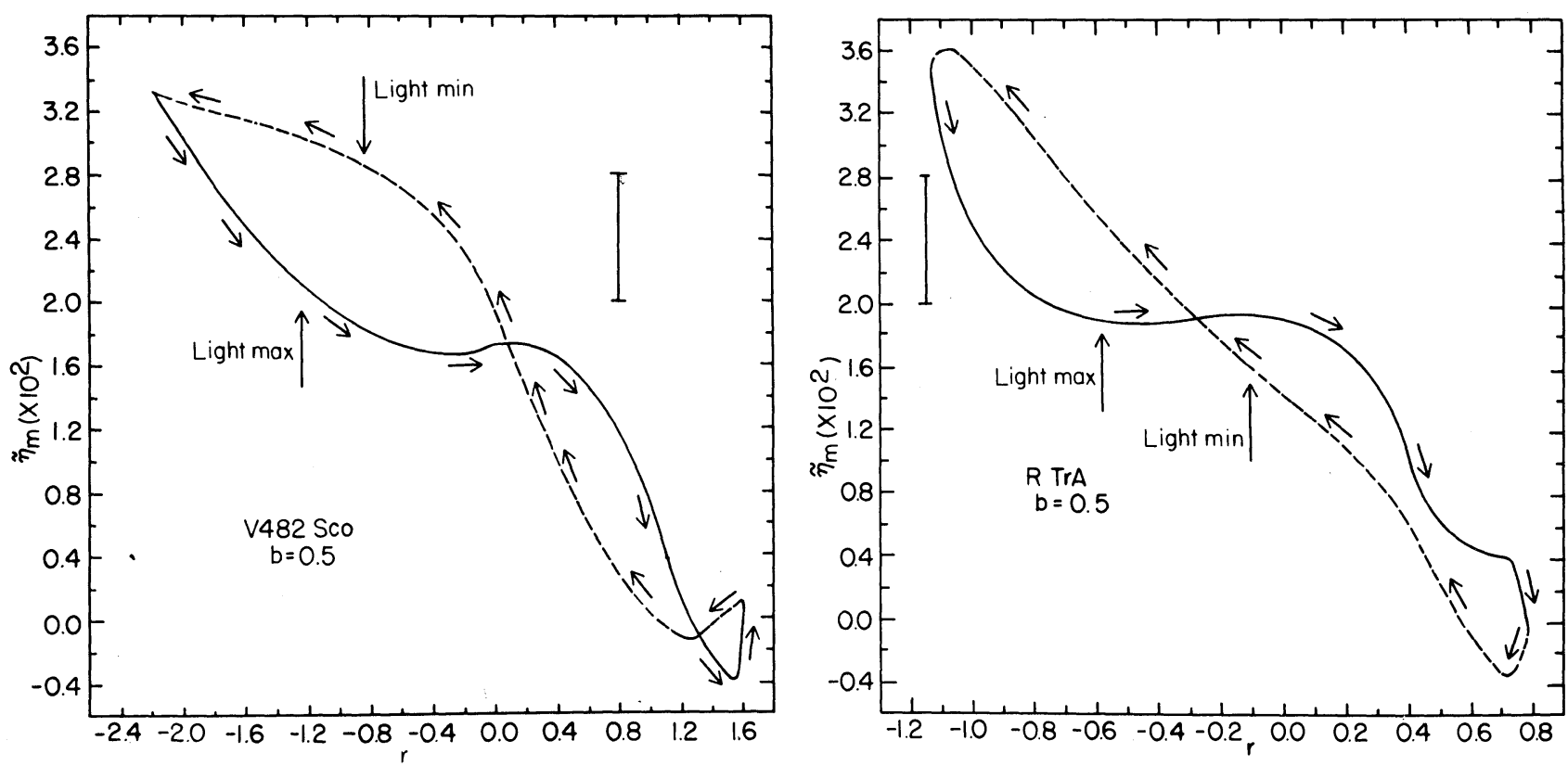

FIG. $2-\tilde{\eta}$ vs. $r$ for the case $b=0.5$. Notation as in Figure 1 . 
negative $\tilde{\eta}_{m}$ has been reduced to a small region near maximum radius; and (3) the loop has narrowed considerably, indicating, once more, that the difference between expansion and contraction has been lessened. Thus it seems clear that a larger value (in this case, $b=0.5$ ) is to be much preferred to $b=0.363$.

It has already been suggested above that some discrepancy is expected in the coefficient due to differences between the Cousins and Johnson systems. To see what role such differences might have played we shall crudely translate the Johnson coefficient $b=0.363$ into a Cousins coefficient $b_{\mathrm{c}}$ as follows. Using the transformation given by Gieren (1984) we calculate for each observational point the value $b_{c}$ necessary to obtain the same surface brightness for the Cousins $(V-R)$ as is calculated for the Johnson $(V-R)$ using $b=0.363$, i.e., $0.363(V-R)_{\mathrm{J}}=$ $b_{\mathrm{c}}(V-R)_{\mathrm{c}}$. We find $0.516 \leq b_{\mathrm{c}} \leq 0.536$ for V482 Sco and $0.529 \leq b_{\mathrm{c}} \leq 0.571$ for $\mathrm{R} \operatorname{TrA}$. These values fall in the same direction as our results above, which makes it seem likely that most or all of the discrepancy is due to the different system.

Nonetheless, the rendition given in Table IV and Figure 2 still leaves much to be desired. From equation (23) it is clear that the loop in the $\tilde{\eta}_{m}$ vs. $r$ diagram ought actually to be compressed to a straight line whose slope would then yield the unperturbed radius, $R_{0}$. While one might justifiably draw such a line with greater confidence in Figure 2, say, than in Figure 1, it remains true that different portions of the loops in Figure 2 indicate quite different slopes. One way of proceeding in this situation would be to find the value of $b$ which minimizes the vertical spread of the loop and then fit a straight line to the diagram which emerges. However, such a method would not take full advantage of the potential of the Baade-Wesselink inversion. We shall suggest an alternative in the section which follows.

\section{A Plan for the Inversion Technique}

The difficulties with the surface-brightness method, pointed out in the previous section, have their origins in the assumption that the temperature and bolometric correction are both linear functions of some given color. Many authors have emphasized the inadequacy of this assumption (e.g., Gautschy 1986; Cohen and Gordon 1987), particularly in the face of the fact that hydrodynamic motions in the pulsating atmosphere are very different during different parts of the cycle. In what follows we shall suggest a modification of the surface-brightness technique which helps meet these objections.

Let us then return to equations (9) and (10) and write

$$
\tilde{R}=F\left(C_{1}, C_{2}, \mathrm{C}_{3}, C_{4}, v, a, s\right),
$$

where the $C_{i}$ are different colors, $v$ is the pulsational velocity, $a$ the acceleration, and $s$ is another quantity obtained from the spectral lines, e.g., the instantaneous equivalent width from the line blend of a spectrophotometer.

Suppose one observes a group of classical Cepheids, obtaining very accurate radial velocities, line widths, and also photometry in the four colors (or six if one chooses) indicated schematically above. These observations may then be used to determine the function $F$; i.e., we propose to parameterize the instantaneous luminosity and temperature in the form $\tilde{R}=L^{1 / 2} / T_{e}^{2}$, with some function which will be valid over the entire pulsation cycle and also from star to star. The observation of a dozen stars, with 25 pairs of points of equal displacement chosen from each, would yield a set of 300 equations of the form

$$
\tilde{\eta}=\log \tilde{\alpha}\left(C_{i}, a, v, s\right)=0 .
$$

This set would then be employed to specify the function $F$. Reddenings would not be necessary so long as $F$ remained linear in the parameters.

There is every reason to believe that such a parameterization would be successful, since the multiple colors would provide considerable detail in characterizing the radiation of the star, while the inclusion of $v, a$, and $s$ would add information on hydrodynamic motions and, perhaps, on the atmospheric density. Preliminary guesses for the form of the function $F$ could be informed by theoretical work on pulsating atmospheres (e.g., Karp 1975).

When a function is found that minimizes the set $|\tilde{\eta}|$, its validity can be further tested by plotting an $\tilde{\eta}_{m}$ vs. $r$ diagram, similar to Figures 1 and 2 , for each star. We note that while formal satisfaction of equation (25) for every pair of equal displacements in the cycle of a given star will result in a single valued $\tilde{\eta}_{\mathrm{m}}$, the actual curve should be not only single valued but also linear. To the extent that this proves true for each star, the form ultimately selected for the function $F$ will be vindicated.

The successful determination of $F$ would have many important consequences. In the first place, the radius of each star could be read simply from the $\tilde{\eta}_{m}$ vs. $r$ plot. Second, the dependence of $F$ on the various physical quantities would provide a solid observational base for improving hydrodynamic model atmospheres. Third, and perhaps most far-reaching, one could then carry the same technique farther to determine the temperatures and ultimately the luminosities of pulsating stars. We discuss this briefly in what follows.

With known $F$ (eq. (24)) the value of $\tilde{\alpha}$ can be calculated for any pair of phases (eq. (9)), in particular for two phases with unequal displacements. Then, from equation (10) we have

$$
\frac{\tilde{T}_{2}}{\tilde{T}_{1}}=\tilde{\mu}=\left(\frac{L_{2}}{L_{1}}\right)^{1 / 4}(\tilde{\alpha})^{1 / 2}
$$


We may now attempt to parameterize $T$ as in equation $(24)$

$$
\tilde{T}=G\left(C_{i}, v, a, s\right),
$$

where the function $G$ can be determined from the set given by equation (26), provided one is able to measure the luminosity ratio for arbitrary pairs of phases. While this is a time-consuming task, the rewards would be great, since once the function $G$ is specified one has very simply

$$
\tilde{L}=a c \pi \tilde{R}^{2} \tilde{T}^{4}
$$

with $\tilde{R}$ and $\tilde{T}$ given by equations (24) and (27), respectively. Determination of the luminosity in this way would, however, require specification of the reddening.

While the techniques necessary to undertake this program are all well in hand, it seems that a new set of observations will be needed. The data for many of Gieren's stars are sparse-there are numerous phase gaps both in radial velocity (Gieren 1981a) and in the various colors (Gieren 1981b). On the other hand, while the extensive BVRI photometry of Moffett and Barnes (1980, 1984) seems well suited for our purposes, the corresponding radial velocities (not yet published) have a standard error of the order $4 \mathrm{~km} \mathrm{sec}^{-1}$ (Barnes 1986, private communication). The effect of such an uncertainty would be to double the extent of the error bars in Figures 1 and 2 , a circumstance which could hardly be tolerated. On the contrary, there exist spectrophotometric techniques with precision of $<1 \mathrm{~km} \mathrm{sec}^{-1}$ (e.g., CORAVEL, see Meylan et al. 1986); this raises the hope of halving the uncertainty in the inversion method.

Thus we propose a new series of simultaneous light and radial-velocity observations of perhaps a dozen classical Cepheids. These observations do not require a large telescope but should be made with state-of-the-art techniques and with care to secure solid phase coverage for each star. With such data in hand, we believe that the inversion of the Baade-Wesselink technique can proceed with enough accuracy that the radii of the classical Cepheids may be pinned down once and for all.

The author is pleased to acknowledge informative discussions with T. J. Moffett, E. G. Schmidt, and T. J. Teays as well as support from the National Science Foundation under grant AST 83-16875.

\section{REFERENCES}

Barnes, T. G. 1980, Highlights of Astronomy, 5, 479.

Barnes, T. G., Dominy, J. F., Evans, D. S., Kelton, P. W., Parsons, S. B., and Stover, R. J. 1977, M.N.R.A.S., 178, 661.

Barnes, T. G., and Moffett, T. J. 1985, in Cepheids: Theory and Observations, ed. B. F. Madore (Cambridge: Cambridge University Press), p. 53.

Cohen, J. G., and Gordon, G. A. 1987, Ap. J., 318, 215.

Gautschy, A. 1986, Astronomical Institute, University of Basel, Preprint Series, No. 23.

Gieren, W. P. 1981a, Ap. J. Suppl., 46, 287. 1981b, Ap. J. Suppl., 47, 315. 1984, Ap. J., 282, 650. 1985, in Cepheids: Theory and Observations, ed. B. F. Madore (Cambridge: Cambridge University Press), p. 38.

Hindsley, R., and Bell, R. A. 1986, Pub. A.S.P., 98, 881.

Karp, A., 1975, Ap. J., 201, 641.

Meylan, G., Burki, G., Rufener, F., Mayor, M., Burnet, M., and Ischi, E. 1986, Astr. Ap. Suppl., 64, 25.

Moffett, T. J., and Barnes, T. G. 1980, Ap. J. Suppl., 44, 427. 1984, Ap. J. Suppl., 55, 389.

Simon, N. R., and Teays, T. J. 1983, Ap. J., 265, 996. 